\title{
4
}

\section{Developing performance networks to improve the benchmarking process - Action Research for Productivity Improvement}

\author{
Thomas A. Hansen and Jens O. Riis \\ The Department of Production, University of Aalborg \\ Fibigerstraede 16, 9220 Aalborg, Denmark
}

\begin{abstract}
Benchmarking is related to many areas of interest when improving the competitiveness of an industrial enterprise or any other activity center which functions under market economic conditions. An important element in gaining full benefit from a benchmarking process is the ability to implement cross functional activities and manage change. This ability may be improved by the implementation of performance networks which are aligned according to tasks and core competences. This paper discusses a framework for implementing performance networks and assessment of the performance network maturity stages, which is important if feasible improvement actions are to be initiated. The paper is based on results from a Danish research project in an industrial enterprise.
\end{abstract}

\section{IMPLICATIONS OF BENCHMARKING}

The benchmarking process may be defined as follows (Camp, 89):

"Benchmarking is the continuous process of measuring products, services, and practices against the toughest competitors or those companies recognized as industry leaders".

This definition holds important implications as word 'continuous' indicates that benchmarking should not be thought of as a one time event. Furthermore the word 'measure' indicates that evaluation of performance based on derived performance measures has to be carried out. Benchmarking activities may be divided into two major groups: internal and external benchmarking. Internal benchmarking is application of the benchmarking philosophy (comparing yourself to the best) within your own organization, i.e. comparing business processes and performance of one organizational units to that of others. It is not only establishing benchmarks (performance measurement). As to external benchmarking one may distinguish between competitive benchmarking which is focused on direct competitors within the same industry and non-competitive benchmarking which is focused on organizations which are not direct competitors. The benchmarking process may be divided into the following steps (from Camp, 89):

Planning (Identify what is to be benchmarked, Identify comparative companies or activity centers, Determine data collection method and collect data).

Analysis (Determine current performance gap, Project future performance levels).

Integration (Communicate benchmark findings and gain acceptance, Establish goals).

Action (Develop action plans, Implement specific actions and monitor progress, Recalibrate benchmarks). 
As discussed in a later paragraph it is important to recognize that most of the activities listed above involves organizational change and that a benchmarking process benefits from dynamic cross functional organization structures.

\subsection{Benchmarking and performance assessment}

Benchmarking implies performance assessment in at least two different environments, your own and the reference object. When benchmarking is used as a leverage technique, it is important to find cardinal competences which may be subject to change and improvement. This may be accomplished by using the model illustrated in figure 1 , in which performance requirements of the market are compared to the specific company performance.

The model may be applied to different activity levels, e.g. company level, function level or production unit level etc. The model essentially enables an illustration of company performance to the market "average" and how the portfolio of selected competences support a continuous competitive strength. When the model is applied it is necessary to select a competence related to one or more of the generic performance areas i.e. features (product) and quality, logistics or productivity (manufacturing). The next steps are to assess the market performance (competition point of view) and the company performance. The market performance is separated in two categories with different characteristics. The first category implies a scale of competences, skills or properties which may be denoted 'Qualifiers'. Qualifiers are the foundation for a competitive strength and 'a must' if long term survival is to be achieved. In addition to qualifying properties a range of innovative capabilities should be possessed, because these are the edge-keeping properties of the company and make out the future competitive profile.

Two categories have been introduced in order to represent the company performance profile. For a skill or competence within any performance area we have chosen to distinguish between those fully possessed and those not possessed. If a skill or competence is possessed it should be mastered to an extent enabling optimal use in business processes or production. A skill or competence which is not mastered must undergo further research, development or implementation and training before it is part of the self propelled business organization.

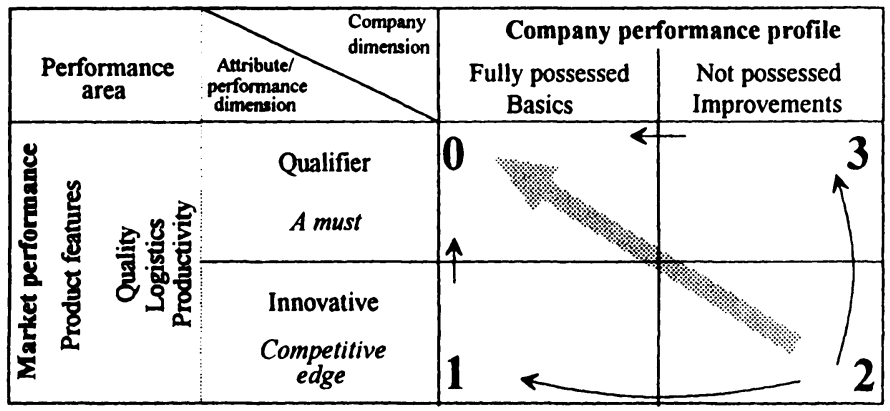

Fig. 1: Capability evaluation model supporting benchmarking efforts and focus.

The different fields of the matrix implies different competitive situations and possibilities. Location in field ' 0 ' means that the company masters a competence (e.g. a process, technology etc.) completely which makes it a basic attribute. This attribute does however not provide you with a specific competitive edge as the industry in general offers these attributes at the same 
performance level. Location in field ' 1 ' is only possible if you have either differentiated attributes to offer or if your performance related to these attributes is superior to your competitors because you have managed to make it a basic competence or property of your company. If not, you will be located in field ' 2 ', which may be perceived as a future competitive option. Location in field ' 3 ' is not desirable, as investments have to be made in order to move from field ' 3 ' to ' 0 '.

The path ' 2 ' --> ' 0 ' is quite neutral, and the one most companies will follow in the long run. The path ' 2 ' to ' 1 ' is followed by world class performers. Time plays an important role in the model as companies continuously slide towards the ' 0 ' field. Thus if the time span going from not possessed to fully possessed is too great it will be very difficult to enter the ' 1 ' field. This is caused by the fact that what is new today is not new tomorrow and the customers will either expect the attribute or a competitor will possess the capabilities necessary to offer it.

The capability evaluation model has proven very useful in the high-level business planning process at a Danish industrial enterprise and is believed to be a helpful processing-tool in relation to the planning and analysis phase of benchmark studies. Further more the model helps illustrate the possible effects of the implementation of performance networks. Performance networks play an important role in relation to performance improvement and change and therefore also supports a successful benchmarking study (esp. the integration and action phases) and also helps companies move directly from field ' 2 ' to field ' 1 '.

\section{INTRODUCING PERFORMANCE NETWORKS}

An organization may be separated into a vertical and a horizontal dimension. The vertical dimension is the most familiar, as investigation of this dimension reveals a typical organization chart with a hierarchical structure limited by functional boundaries. The horizontal dimension appears when a flow-oriented viewpoint is taken. Such a viewpoint reveals the many tasks which cut across functional boundaries such as planning, development etc. This viewpoint enables a new organization chart to be drawn, as the organization members are related to each other by tasks and cross functional objectives.

A good benchmarking study often reveals potential improvement areas and therefore results in change activities. A good mental picture may be a 'change bomb'. It is not likely that the full effect of this change bomb will be obtained unless organizational boundaries are not loosened. A fox-hole organization (cfr. Savage, 93) with stiff functional boundaries would minimize the benefits of a benchmarking process as the shock waves are not allowed to spread and give other organizational units an opportunity to initiate change. The horizontal dimension must be perceived and used pro-actively.

The idea of performance networks is based on combining theory and practice of performance measurement and organizational networking (Hastings, 93). The results are characteristics of a work-form and some tools centered around performance assessment enhancing implementation of a change oriented organization focused on results and cross functional tasks.

\subsection{Defining performance networks}

Numerous researchers, consultants and authors interested in organizational development have discussed the perspectives of networks and organizations. It seems however that it is extremely difficult to find a definition of the term including a specification of what the necessary means and conditions for establishment of a networking organization are. Some authors come close to a definition (esp. Hastings, Savage (1993)), but never realizes concrete suggestions. In order to carry out a stringent discussion, a work-definition of performance networks is proposed and an investigation of how performance networks should be and are established will be initiated. 
A performance network is an organizational cluster established according to recognized cross functional objectives or interdependencies and which aligns improvement efforts to shared and task oriented performance measures.

Performance networks are intended to work cross functionally taking a starting point in interdependencies and interactions (symptoms of cross functional malfunctioning). The basic ideas is that such a starting point is a foundation for analysis and perception of the complex horizontal flow and to identify crucial customer-client relationships. Perception of the horizontal flow and organization enables co-operation and establishment of performance measures focusing on the horizontal organization.

However most organizations are not very good at realizing true horizontal performance networks. Performance measurement has traditionally been very vertically focused. The primary reasons are the complexity of the horizontal system and the traditional functional separation of responsibility. The vertical focus however stimulates sub-optimation and hinders integration. Therefore horizontal performance measurement is a crucial focal point of productivity improvement of the future.

From a theoretical viewpoint, a model implying concurrent development and exploitation would be more suitable, but from an applied viewpoint, taking into account the performance measurement state of the art in most industries, it is suggested that the evolution of performance measurement should progress towards the horizontal dimension by climbing the "Performance Measurement Ladder" shown in figure 2.

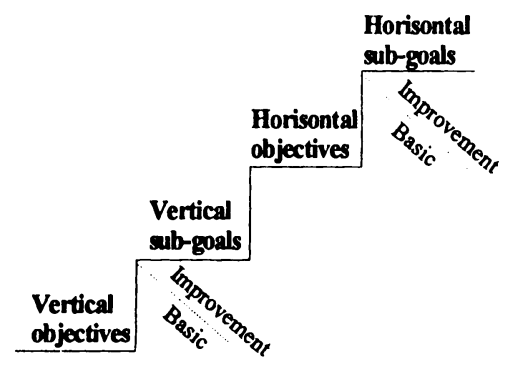

Fig. 2: The performance network ladder

The levels of the performance measurement ladder actually describe the development of a certain work-form along with the establishment of certain structural conditions. The nature of the work form and the structural conditions are described in the following. The implications of the performance network ladder are founded on well known constructs and have been verified during action research at an industrial enterprise.

\subsection{Characterizing the levels of the performance ladder}

Two factors are important to discuss in relation to performance networks: 1) How tasks and responsibility are defined and handled, 2) How follow-up is established and handled. Each level may be characterized from two viewpoints: a behavioural and a structural. The need for both viewpoints is becoming more and more apparent, as researchers investigate integrated productivity improvement and how implementation of new technology should include parallel work on organizational aspects. 


\subsection{The level of vertical objectives}

\section{Behavioural characteristics}

- Functional borders limit task definition and perspective.

- Objectives are received from functional superior but not really decomposed.

Structural conditions

- Performance measures are established within functional borders at responsibility/task level.

- Follow up is initiated by the superior and targets the mass or responsible individuals.

\subsection{The level of vertical sub-goals}

\section{Behavioural characteristics}

- Functional borders limit task definition and perspective

- Objectives received from functional superior are decomposed and operationalized.

- Analysis are to a large extent initiated and carried out by organization members themselves.

Structural conditions

- Performance measures are established within functional borders at sub-task level by organization members themselves.

- Follow up is initiated by organization members and targets sub-task owners.

\subsection{The level of horizontal objectives}

\section{Behavioural characteristics}

- Functional borders are recognized but does not limit cross functional task definition.

- Horizontal objectives are identified and established but not decomposed.

Structural conditions

- Performance measures are established at task level.

- Horizontal follow up is initiated by superior and targets the mass or the responsible individuals.

\subsection{The level of horizontal sub-goals}

\section{Behavioural characteristics}

- Cross functional task definition is widespread, and constantly initiated by organizational members themselves.

- Decomposition of horizontal objectives by organization members themselves

- Cross functional analysis are carried out by task-owners themselves.

Structural conditions

- Performance measures are established at task level but supported by operational sub-task measures established by organizational members themselves.

- Follow up is initiated by organization members and targets sub-task owners.

In todays organizations most units are found on the vertical 'steps'. It seems that two primary reasons influence this: 1) The vertical view of the organization is traditional and easy to cope with. 2) The organizational structures and the management systems (IT) are geared to function in a vertical environment. It is important to seek a two-dimensionally balanced organization where employees are influenced not only to look in a vertical direction but also to work actively in a 
horizontal dimension which includes participation in decomposition of horizontal objectives and in developing management systems that take these issues into account.

\subsection{Differentiating between basics and improvements}

In the performance network ladder (fig. 2) it is indicated that there is a need to differentiate between the basic dimension (already possessed tasks or competences) from the improvement dimension. This will allow a continuous improvement process to be initiated and structured. Further discussion of this argument and how it may be combined with the network establishment may be found in a later paragraph.

\subsection{Implications of performance networks}

Horizontal performance networks implies a measurement system (collection of measures) centered around the horizontal task structure. The purpose of the measurement system is to focus attention to whatever activities or evaluation criteria are important in order to generate improvement of productivity and performance. According to the theory on development of measures and measurement systems, a number of behavioural and structural considerations are to be investigate.

Development of measures should be based on situational analysis and knowledge of the desired relationship between objectives, means (decision variables) and the measurement system itself.

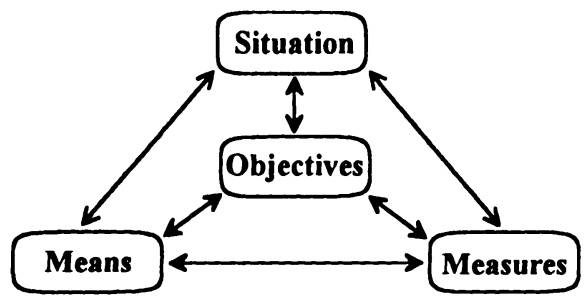

Fig. 3: Central elements of performance measurement and improvement.

Development of a measurement system often will include the following activities: 1 . Decomposition of objectives, 2. Analysis of objectives and means, 3. Construction of measures, 4. Implementation of measures and measurement procedures. 5. Development of information and documentation procedures. Experience from applied measurement system elaboration have shown that success is supported heavily by initiating a participary and costumer driven development process (Bitton, $90 \&$ Brinkerhof, 90). Further more the measures developed and established should (cfr. Hansen, 93 \& Christopher, 93):

1. Be accepted by employees concerned with performance measurement and evaluation (this usually means that they have participated in the development and establishment process).

2. Be established in accordance with the objectives of the system studied.

3. Be easily measured, easily quantified and easily understood.

4. Be situationally adapted in frequency and aggregation.

5. Allow coherence between long term and short term objectives.

If the idea of performance networks and horizontal performance measurement is combined with the knowledge and implications of theory behind performance measurement, a large number 
of complex questions arise. The questions are easy to identify if the behavioural and structural characteristics of the horizontal sub-goal level mentioned earlier are reviewed. Some of them are:

How may the horizontal dimension be assessed.

- How are task (cross functional) perspectives captured, analyzed and visualized

- How are shared and consistent performance measures established, decomposed an implemented.

How are relevant performance networks identified and established.

- How may information technology be used to support performance networks.

Investigation of these questions is relevant as it often is the basis of performance evaluation in the organization. Thus actions and organizational values are highly influenced by the measurement system. Some tools and considerations concerning establishment of performance networks through performance measurement are presented in the following paragraph.

\section{STRUCTURING MEASUREMENT AND IMPROVEMENT EFFORTS}

It has been argued that the initial phases of a benchmarking process (planning and analysis) may be supported by applying the model and the underlying mind set of figure 1. This paragraph discusses another model which may support the phases 'Integration' and 'Action' of the benchmarking process and help organizational members establish performance networks by climbing the performance measurement ladder.

\subsection{Enabling the organization}

The implications of horizontal performance measurement are often very demanding to organizational members, as they are asked to bust boundaries and seek partners outside their own domain. Therefore it is important to provide tools to help them structure the efforts and obtain success. The model in figure 4 is meant to help balance performance improvement in both the vertical and horizontal dimensions and to structure performance measurement. The model has been used in practice with much success in pilot projects at one company, and will be an important instrument in the quest of reaching the upper levels in the performance measurement ladder throughout the organization. The model is extremely simple and may therefore be used by all organizational members.

The model is based on distinguishing between the vertical and horizontal dimension and also between performance areas or measures which focuses on basic activities as opposed to improvement activities. The separation between a vertical and horizontal dimension has already been discussed. The basics are the performance areas or tasks which are mastered well, indicating a good balance between the four elements of figure 3 (situational boundaries, objectives, means and measures). The improvements are performance areas or tasks which are identified but not mastered.

The model enables a continuous and structured increase in performance as organizational members are motivated to force an increasing number of tasks and performance areas into the fields of 'Basics'.

The general patterns of how an organizational unit is placed in the model are somewhat given by the performance measurement ladder. This results in a pattern where organizational units studied often move from working in field ' 0 ' or ' 1 ' and gradually progress towards field ' 3 '. Operating in field ' 3 ' means dealing with and assessing complex problems in a wholistic manner. 


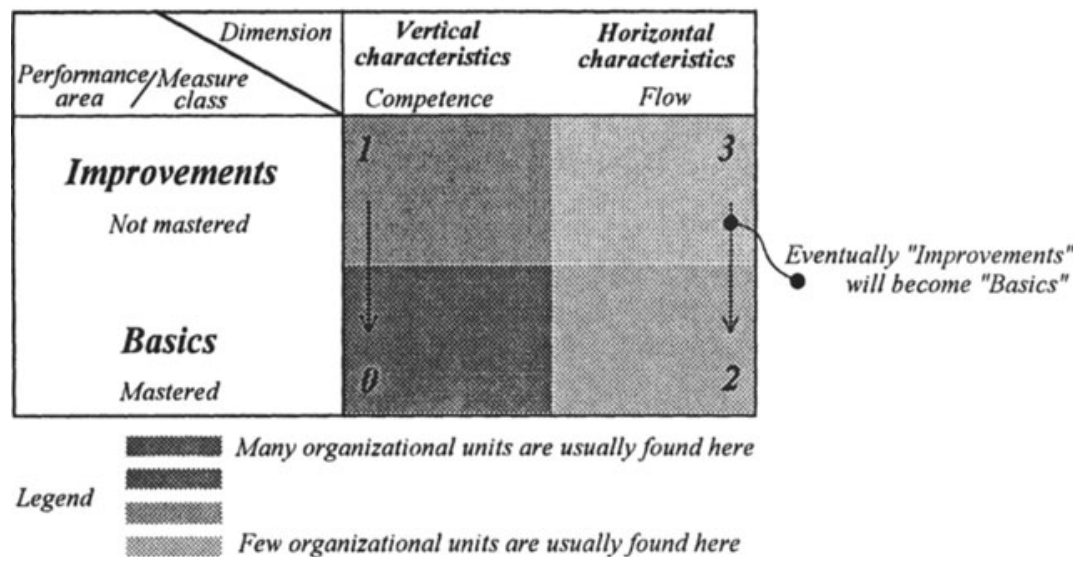

Fig. 4: Generic model for structuring measurements and improvement efforts.

As discussed later, it is possible to evaluate where an organizational unit currently is located. The model has proven itself extremely useful when implementing performance networks in specific production units, but has also been appreciated at higher organizational levels as a cognitive tool.

At higher organizational levels the model was applied in an effort to map the activity span and its characteristics. The objective was to initiate a discussion whether or not the initiatives launched were balanced correctly (as desired) in the different fields of the model. Thus the model was given substance by discussing the characteristics of the results expected given the situation and use of certain means (in terms of basic/improvement, vertical/horizontal).

At lower organizational levels (production units and sections) the model was applied during an effort where the main objective was to structure the forthcoming improvement efforts in a manner that invited to analyze across functions, find partners and establish shared performance measures. Thus a focus was on tasks and appropriate performance measures and their location in the different field of the model. This way a moderate but steady expansion of tasks and performance areas was initiated. At the same time a new way of operation focusing on horizontal dimensions began to appear.

\subsection{On the need of horizontally oriented analysis methods}

As performance networks are based on horizontal performance measures it is clear that need for cross functional analysis and co-operation appear. However this means that tools supporting these efforts must be developed. When the need of analysis methods is considered, the following points should be kept in mind:

- Employees influenced by the performance measures and the following evaluation should participate in developing and establishing the performance measures needed in order for the measures to be accepted. Final commitment may only be accomplished if employees sense that they have access to some active decision variables which enable them to influence the performance level. 
- Organizations are becoming leaner which means that fewer managers and advisers are available in the effort to carry out horizontal analysis. Employees will have to (and should) participate in the analysis effort.

There are major research areas related to these considerations. Important ones are performance measurement systems, consistency analysis, modelling of relationships and interdependencies including the use of information technology (IT) supporting the areas mentioned.

Without going into methodological details, many of the analytical methods and tools known today are most often used consequently by researchers and consultants, and more seldomly managers or operators close to the shop floor. Examples are application of IDEF/SADT diagraming, concept development, activity chain analysis. Even the 'simple' formalisms, tools and techniques usually embraced by the Kaizen-term have been very difficult to implement or effectuate in numerous industrial enterprises in Europe. Thus if a self propelled organization is to be developed there is a need to develop methods and tools useful for horizontal analysis which are simple and yet powerful.

\section{THE PERFORMANCE NETWORK MATURITY LEVEL}

In several cases during the research project it has been useful to apply evaluation principles given by Kirkpatrick (Kirkpatrick, 77) in order to seek an understanding of the current maturity level of the organizational unit studied. The maturity level analysis takes a starting point in the different levels of the performance network ladder and their characteristics. Applying these principles results in an approach illustrated in fig 5.

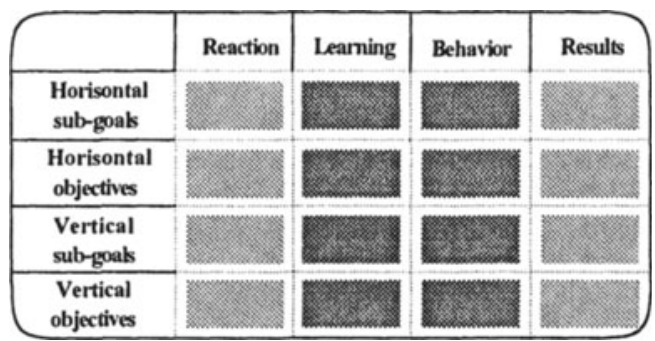

Fig. 5: Organizational performance network maturity assessment

The basic idea of the evaluation model is to assess the organization by studying selected units at four different evaluation levels: 1)Reaction, where the objective is to investigate the reactions of the different participants involved when exposed to the ideas and implications of performance networks as presented in this paper. 2)Learning, where the objective is to obtain information of the knowledge, skills and attitudes of the participants involved when exposed to the ideas and implications of performance networks. 3)Behaviour, where the objective is to obtain information concerning the behaviour of the participants involved. This makes a comparison of desired and actual behaviour possible. 4)Results, where the objective is to investigate how results are influenced by existing behavioural characteristics and established structural conditions.

It is important that the investigation targets all evaluation levels of the model shown in figure 5 , but experience applying the model has led to the conclusion that the learning and behavioural levels are crucial, as much useful information may be obtained if those levels are assessed correctly. 
By evaluating organizational units using the model in figure 5 , it is possible to find many signals indicating a feasible path in order to implement performance networks in an industrial enterprise. This has been done in the research project, and both interviews and questionnaires have been applied with much success. The result was development of project ideas and action plans along with initiating a learning process within the units studied.

\section{CONCLUSIONS}

Benchmarking may support companies in the effort to improve their competitive situation. A benchmarking study is however often centered around a specific organizational unit and a selected business process. The potential benefits of benchmarking increase if the organizational processes in a benchmarking study is not limited by stiff functional boundaries. It is important that the benchmarking process is related to the implementation of horizontal relations as unrealized competitive strength may be found here.

Horizontal relations and interdependencies are central elements in developing performance networks within an organization. This paper discussed why and how performance networks should be established and argued that application of benchmarking along with implementation of performance networks may catalyse realizing the competitive possibilities found in well functioning horizontal relations of any company or corporation. A number of models and tools developed and tested during work with performance networks in an industrial enterprise have been presented.

\section{REFERENCES}

Bitton, Marc: ECOGRAI, Méthode de Conception et d'Implementation de Systemes de Mesure de Performance pour Organisations Industrielles. Thèse Docteur, Université de Bordeaux, Septembre 1990.

Brinkerhoff, Robert O. \& Dennis E. Dressler: Productivity Measurement - A Guide for Managers and Evaluators. Applied Social Research Methods Series, Vol. 19. Sage Publications, 1990.

Chew, W.Bruce, Timothy F. Bresnahan, Kim B. Clark: Measurement, Coordination, and Learning in a Multiplant Network. Measures for manufacturing Excellence edited by Robert Kaplan, Harvard Business School Series in Accounting and Control, 1990.

Camp, Robert C.: Benchmarking - The search for industry best practices that lead to superior performance. Quality Press, Milwaukee, Wisconsin, 1989.

Christopher, William F. (ed.) \& Carl G. Thor, (ed.): Handbook for Productivity Measurement and Improvement. Productivity Press, Massachusetts, 1993.

Frick, Jan \& Jens O. Riis: Activity Chains as a Tool for Integrating Industrial Enterprises. Advances in Production Management Systems. Proceedings of the 4th IFIP/TC5/WG5.7 APMS'90 Conference, North-Holland 1991.

Hansen, Thomas A. \& Jens O. Riis: Modelling Interactions and Measuring Performance for Productivity Improvement. Proceedings of the 8th IPS Research Seminar, 1993. Report from Dept. of production, University of Aalborg, 1993.

Hastings, Colin: The New Organization - Growing the culture of organizational networking. McGraw-Hill International (UK) Limited, 1993.

Kirkpatrick, Donald L.: Evaluating Training Programs: Evidence vs. Proof. Training and Development Journal, November 1977.

Savage, Charles M.: $5^{\text {th }}$ Generation Management - Integrating Enterprises through Human Networking. Digital Press, 1990. 